\title{
Duração do sono, excesso de peso e consumo de alimentos ultraprocessados em adolescentes
}

\author{
Sleep duration, overweight and consumption of ultra-processed \\ foods among adolescents
}

Elaine Valdna Oliveira dos Santos (https://orcid.org/0000-0002-1808-1385) ${ }^{1}$ Aléssio Tony Cavalcanti de Almeida (https://orcid.org/0000-0003-0436-359X) ${ }^{2}$ Flávia Emília Leite de Lima Ferreira ( http://orcid.org/0000-0002-2849-9902) ${ }^{3}$
${ }^{1}$ Centro de Educação e Saúde, Universidade Federal de Campina Grande. Sítio Olho d'água da bica s/n, Olho d'água. 58175000

Cuité PB Brasil. nut. elaineoliveira@gmail.com ${ }^{2}$ Departamento de Economia, Universidade Federal da Paraíba (UFPB). João Pessoa PB Brasil. ${ }^{3}$ Departamento de Nutrição, UFPB. João Pessoa PB Brasil.

\begin{abstract}
The scope of this article is to analyze the relationship between insufficient sleep duration, overweight/obesity and the consumption of ultra-processed foods among adolescents aged 10 to 14 years. This is a cross-sectional study, with an evaluation of 1,384 adolescents from public schools in João Pessoa-PB, participating in the Longitudinal Study on Sedentary Behavior, Physical Activity, Diet and Adolescent Health (LONCAAFS). Sociodemographic variables, sleep duration, class shift, anthropometric nutritional status, sedentary behavior and food consumption were measured. Linear and logistic regression of the following were performed using Stata 13.0 Software: the prevalence of short sleep duration of $29.5 \%$ ( $<9 \mathrm{~h} /$ night $)$; a significant association between short sleep duration and excess weight only for adolescents $<12$ years old. With respect to the relationship between sleep duration and the consumption of ultra-processed foods, the longer the sleep duration, the lower the consumption of snacks by adolescents under 12 years old, with a positive association for $\geq 12$ years old only with adjustment by the physical activity variable. There was no association with the "sugary drinks" and "cookies" groups for any of the age groups analyzed.
\end{abstract}

Key words Sleep, Adolescent, Overweight, Obesity, Food consumption
Resumo $O$ objetivo deste artigo é analisar a relação entre duração insuficiente do sono, sobrepeso/obesidade e o consumo de alimentos ultraprocessados em adolescentes de 10 a 14 anos. Trata-se de um estudo transversal, com avaliação de 1.384 adolescentes de escolas públicas de João Pessoa-PB, participantes do Estudo Longitudinal sobre Comportamento Sedentário, Atividade Física, Hábitos Alimentares e Saúde de Adolescentes (LONCAAFS). Foram mensuradas variáveis sociodemográficas, duração do sono, turno de aula, estado nutricional antropométrico, comportamento sedentário e consumo alimentar. Foram realizadas regressão linear e logística por meio do Software Stata 13.0. Prevalência de curta duração do sono de 29,5\% ( $<9 \mathrm{~h} /$ noite). Associação signifcativa entre a curta duração do sono e o excesso de peso somente para adolescentes $<12$ anos. Quanto à relação entre a duração do sono e o consumo de alimentos ultraprocessados, quanto maior a duração do sono, menor o consumo de lanches pelos adolescentes menores de 12 anos, com associação positiva para $\geq 12$ anos apenas com ajuste pela variável atividade física. Não houve associação com os grupos "bebidas açucaradas" e "biscoitos" para nenhuma das faixas etárias analisadas.

Palavras-chave Sono, Adolescente, Sobrepeso, Obesidade, Consumo de alimentos 


\section{Introdução}

O sono é um processo fisiológico originado pela combinação de mecanismos homeostáticos e cronobiológicos, a partir de fatores que incluem determinantes biológicos, cognitivos, culturais e ambientais ${ }^{1-3}$, com provável influência no estado nutricional antropométrico de crianças e adolescentes $^{4-6}$.

Um sono adequado na adolescência relaciona-se ao crescimento e desenvolvimento físico, mas também emocional e comportamental ${ }^{2,7}$. A National Sleep Foundation ${ }^{8}$ recomenda que indivíduos de 6 a 13 anos de idade durmam de 9 a 11 horas por noite, e que aqueles de 14 a 17 anos durmam de 8 a 10 horas.

A curta duração do sono vem sendo investigada dentre as variáveis que são potenciais influenciadores do excesso de peso em adolescentes, por possuir grande contribuição na modulação do metabolismo e função neuroendócrina dos mesmos ${ }^{9,10}$. Sabe-se que o sobrepeso pode evoluir para obesidade, e favorecer as desordens metabólicas que podem ser influenciadas pela rotina de sono ${ }^{11}$, devendo haver atenção especial para a falta de prática rotineira de atividades físicas, tempo em comportamento sedentário e consumo insuficiente de alimentos saudáveis como fatores determinantes ${ }^{12}$.

O aumento da ingestão alimentar após restrição de sono pode ocorrer mesmo não havendo alterações hormonais, pois o fato de permanecer acordado por mais tempo dá ao adolescente a oportunidade para alimentar-se e, geralmente, a partir de alimentos calóricos, muitas vezes ricos em gorduras ${ }^{13}$.

De acordo com metanálise realizada recentemente ${ }^{14}$, uma curta duração do sono está relacionada a um maior consumo de refrigerantes e lanches em crianças e adolescentes. Alguns estudos fortalecem ainda a hipótese de que a má qualidade da dieta em decorrência da privação de sono pode estar relacionada ao surgimento de obesidade $^{10,15-17}$.

Considerando o aumento da prevalência de sobrepeso e obesidade em adolescentes, como também do consumo de ultraprocessados, e diante da necessidade de atuar na prevenção da obesidade a partir de seus fatores de risco, o objetivo deste trabalho foi analisar a relação entre duração insuficiente do sono, sobrepeso/obesidade em adolescentes e o consumo de alimentos processados.

\section{Métodos}

Trata-se de um estudo transversal, de base escolar, no qual foram avaliados 1.438 adolescentes de ambos os sexos, com idade mínima de 10 e máxima de 14 anos, matriculados no $6^{\circ}$ ano do ensino Fundamental II de escolas públicas (municipais e estaduais) do município de João Pessoa-PB, participantes da linha de base do Estudo Longitudinal sobre Comportamento Sedentário, Atividade Física, Hábitos Alimentares e Saúde de Adolescentes (LONCAAFS).

O projeto foi aprovado pelo Comitê de Ética em Pesquisa com Seres Humanos do Centro de Ciências da Saúde (CCS) da Universidade Federal da Paraíba (UFPB). O consentimento informado foi obtido dos responsáveis por todos os adolescentes que concordaram em participar.

Para o cálculo de tamanho da amostra considerou-se tamanho da população de referência igual a 9.520 adolescentes, prevalência do desfecho igual a $50 \%$, intervalo de confiança de $95 \%$, erro absoluto aceitável de $4 \%$ e efeito de desenho (deff) igual a 2. O tamanho mínimo da amostra foi estabelecido em 1.130 adolescentes, com acréscimo de $40 \%$ a fim de compensar perdas e recusas, resultando em amostra de 1.582 adolescentes.

A amostra foi composta por adolescentes de 28 escolas, selecionadas sistematicamente por conglomerados em estágio único (14 municipais e 14 estaduais) distribuídas proporcionalmente conforme tamanho (número de alunos matriculados no $6^{\circ}$ ano) e região geográfica no município (norte, sul, leste, oeste). Todos os alunos de $6^{\circ}$ ano foram convidados a participar do estudo.

A coleta de dados ocorreu entre março e dezembro de 2014 e foi realizada por meio de entrevista face a face, em questionário organizado em blocos temáticos (variáveis sociodemográficas, turno de aula, avaliação do sono, comportamentos sedentários, avaliação nutricional antropométrica e consumo alimentar) e testado anteriormente em estudo piloto. Todos os entrevistadores foram treinados previamente para a padronização da coleta.

Foram excluídos adolescentes abaixo de 10 e acima de 14 anos de idade, com alguma deficiência que impedisse ou limitasse a entrevista; grávidas ou aqueles que não apresentavam dados referentes à duração do sono, medidas antropométricas ou consumo alimentar ou participantes com relato de ingestão energética total diária inferior a $500 \mathrm{kcal}$ ou superior a $6000 \mathrm{kcal}^{18}$. 
Foram avaliados sexo (masculino/feminino), idade (em anos completos), escolaridade da mãe (até ensino fundamental incompleto/até ensino médio incompleto; ensino médio completo ou mais anos) e classe econômica (segundo classificação da Associação Brasileira de Empresas de Pesquisa ${ }^{19}$.

O turno de aula foi referido pelos adolescentes como manhã, tarde ou integral. Nas escolas públicas de João Pessoa o turno da manhã inicia-se às $7 \mathrm{~h}$ e estende-se até as $12 \mathrm{~h} 30$. As aulas da tarde começam às $13 \mathrm{~h}$ e vão até as $17 \mathrm{~h} 30$. E para os alunos que estudam durante o turno integral, as atividades na escola são iniciadas às $7 \mathrm{~h}$, com término às $17 \mathrm{~h} 30$.

A restrição do sono foi observada a partir do questionamento dos horários de dormir e acordar tanto nos dias com aula quanto nos fins de semana, com as seguintes perguntas: "Em um dia normal de semana (segunda a sexta-feira) que horas você dorme? Acorda?"; "Em um dia normal de fim de semana (sábado ou domingo) que horas você dorme? Acorda?". Após a coleta dos dados, foi realizada a média ponderada de tempo de sono por dia, calculada a partir da seguinte equação: [(noites de aula x 5) + (noites de fim de semana $\times 2) / 7]^{17}$, com curta duração do sono referente a menos de 9 horas de sono por noite ${ }^{20}$.

A qualidade do sono foi avaliada a partir da questão "De maneira geral, como você avalia a qualidade do seu sono?”. Após o questionamento, os adolescentes eram apresentados às seguintes opções de resposta: ruim, regular, boa, muito boa e excelente. Para análise a variável foi recategorizada em qualidade de sono negativa (ruim e regular) e positiva (boa, muito boa, excelente).

A variável comportamento sedentário consistiu em perguntas sobre atividades realizadas em posição sentada/reclinada, como assistir televisão e DVD; usar computadores, celulares, tablets e jogar videogames, relatando o número de dias em que realizaram as atividades nos sete dias anteriores à entrevista, bem como o número de horas de uso por dia relatado. Para o cálculo do tempo total de comportamento sedentário, foi feito o somatório do tempo gasto em cada equipamento e o resultado final foi dividido por sete para fornecer o número médio de horas por dia de comportamento sedentário. O comportamento sedentário foi considerado como o tempo gasto sentado/reclinado $\geq 120 \mathrm{~min} / \mathrm{dia}^{21}$.

Para obtenção de dados referentes à atividade física dos adolescentes foi utilizado questionário validado por Farias Junior ${ }^{22}$ para essa população. Foram contabilizados frequência (dias/semana) e duração (minutos/dia) de atividades físicas praticadas por pelo menos 10 minutos na semana anterior à coleta de dados (atividades de intensidade moderada a vigorosa). A partir do somatório dos produtos das frequências pelos tempos de prática em cada atividade foi determinado o tempo total de atividade física (minutos/semana). Foram classificados como fisicamente ativos aqueles com prática igual ou superior a $300 \mathrm{mi}-$ nutos por semana, e os demais como fisicamente inativos ${ }^{23}$.

As medidas antropométricas foram realizadas nas escolas, em local disponibilizado pela direção, livre de rodapés, e onde fosse possível a disposição dos equipamentos necessários à avaliação antropométrica. Para a medida da massa corporal utilizou-se uma balança digital, da marca Bioland ${ }^{\circledR}$, com precisão de 100 gramas, pesando-se o adolescente em posição ortostática, de frente para a parede com os braços paralelos ao corpo. Verificou-se a medida da estatura com um estadiômetro portátil da marca Sanny ${ }^{\circledR}$. Todas as medidas foram realizadas seguindo-se a padronização descrita por Lohman et al. ${ }^{24}$. O diagnóstico do estado nutricional antropométrico foi feito a partir do IMC (massa corporal $[\mathrm{kg}] /$ estatura $\left.[\mathrm{m}]^{2}\right) /$ idade, classificado seguindo os critérios sugeridos pela WHO, segundo idade e sexo, com sobrepeso definido como superior ao percentil 85 e obesidade superior ao percentil $95^{25}$. Todas as medidas foram realizadas em duplicata, sendo realizada uma terceira medida quando a segunda não era igual à primeira.

O consumo alimentar foi obtido por meio do Recordatório de 24h (R24h), aplicado duas vezes, sendo o primeiro em toda a amostra e o segundo em $30 \%$ a fim de diminuir a variabilidade intrapessoal da dieta e aumentar a precisão dos dado ${ }^{26}$. A técnica utilizada para aplicação do inquérito foi a Multiple Pass Method (MPM) ${ }^{27}$. Para auxílio das medições foram utilizados álbuns fotográficos com imagens de medidas caseiras padrão e porções de alimentos cuja mensuração é mais complexa de ser feita ${ }^{28}$.

Os dados de consumo alimentar obtidos por meio dos R24h foram tabulados e processados no software online Virtual Nutri Plus ${ }^{\circledR}$. Posteriormente os alimentos foram selecionados por meio da NOVA classificação que divide os alimentos de acordo com seu grau de processamento industrial, classificando-os em: "alimentos in natura ou minimamente processados", "ingredientes culinários processados", "alimentos processados" e "alimentos ultraprocessados" ${ }^{29,30}$. Os alimentos ultraprocessados foram separados e categoriza- 
dos em grupos: Bebidas açucaradas (refrigerante, suco de caixinha, suco em pó, água de coco em caixinha, xaropes de guaraná/groselha e suco de fruta com adição de açúcar), Biscoitos (Biscoitos recheados e biscoitos salgados) e Lanches (salgadinhos de pacote, salgados fritos, salgados assados e pizzas, macarrão instantâneo, balas, pirulitos, chiclete, caramelo, gelatina).

Para analisar o consumo alimentar foi realizado ajuste da variabilidade intraindividual pelo programa Multiple Source Method (MSM) ${ }^{31}$. O MSM calcula a ingestão usual de indivíduos e grupos, utilizando-se tanto de R24h e registros alimentares quanto questionários de frequência alimentar e compreende três passos de análise: primeiramente é calculada a probabilidade de consumo do alimento em um dia para cada indivíduo. Posteriormente é estimada a quantidade de alimentos consumidos nos dias coletados. Por fim, a ingestão usual é calculada multiplicando-se a probabilidade de consumo de um alimento com uma quantidade usual pelos dias de consumo.

Após a coleta e digitação dos dados, todos os alunos receberam um relatório individual com dados antropométricos, classificação do estado nutricional, e recomendações sobre hábitos alimentares saudáveis, a partir dos resultados coletados nos seus recordatórios. Cada escola recebeu um resumo dos dados dos seus alunos, em forma de gráficos e tabelas, sobre todos os dados coletados.

Para a caracterização da amostra foi realizada estatística descritiva, estratificada pelo número de horas de sono dividido em categorias. A variável sono foi categorizada em curta duração do sono $\operatorname{sim}(<9 \mathrm{~h})$ ou não $(\geq 9 \mathrm{~h})$, com base nas recomendações da National Sleep Foundation (NSF) ${ }^{8}$ para adolescentes. Para o cálculo da associação entre as variáveis categóricas foi realizado o Teste Qui-Quadrado ( $\chi 2)$. A relação entre a duração do sono (variável independente) e o excesso de peso (variável dependente) dos adolescentes foi avaliada por regressão logística, por meio de análise bruta e também ajustada pelas possíveis variáveis de confusão.

Modelos de regressão linear foram realizados tomando-se como variável dependente grupos de alimentos (lanches, bebidas açucaradas e biscoitos) e a duração do sono como variável independente. Todas as análises foram realizadas estratificadas por idade ( $<12$ e $\geq 12$ anos) por meio do software STATA 14.0, utilizando-se o comando Survey, para análises de dados com amostragem complexa. A significância estatística foi estabelecida em $\mathrm{p}<0,05$.

\section{Resultados}

A amostra foi composta por 1.384 adolescentes que apresentavam dados completos de duração do sono, consumo alimentar, antropometria e dados sociodemográficos e cujo relato de ingestão energética total diária era $\geq 500 \mathrm{kcal}$ e $\leq 6000$ $\mathrm{kcal}$. A faixa etária foi compreendida entre $10 \mathrm{e}$ 14 anos, sendo $54 \%$ do sexo feminino (748 adolescentes).

A média de idade foi 12 anos e 40,5\% referiram escolaridade da mãe até ensino fundamental incompleto. A média de duração do sono dos adolescentes foi de 9,6 horas, com curta duração $(<9 \mathrm{~h})$ presente em $29,5 \%$ da amostra.

O consumo médio de calorias foi de 2226.9 $\mathrm{kcal} /$ dia, sendo $706,4 \mathrm{kcal}$ provenientes dos alimentos ultraprocessados $(31,7 \%)$. Com relação ao estado nutricional antropométrico dos adolescentes avaliados, $32,3 \%$ da amostra apresentava sobrepeso ou obesidade.

As características gerais dos adolescentes de acordo com a duração do sono estão descritas na Tabela 1, havendo diferenças estatisticamente significativas apenas para a variável turno de aula $(\mathrm{p}<0,001)$. Dos adolescentes participantes do estudo, $46,5 \%$ dos que estudavam pela manhã e $15,9 \%$ dos que estudavam no turno da tarde apresentaram curta duração do sono. Quando estratificados por sexo, os valores de curta duração do sono correspondem a 28,5\% e 30,7\% para sexo feminino e masculino, respectivamente.

A Tabela 2 mostra o efeito da duração do sono no estado nutricional de adolescentes por meio das análises bruta e ajustada, estratificadas por idade. Observando-se o Modelo I, correspondente à análise bruta, houve efeito significativo, com $\mathrm{OR}=1,61 \quad(\mathrm{IC}=1,01-2,55)$ para menores de 12 anos. Após ajuste pelas variáveis de confusão sexo, turno de aula e qualidade do sono, os valores permaneceram significativos para essa faixa etária (Modelo II: $\mathrm{OR}=1,77$; $\mathrm{IC}=1,09-2,87)$, assim como com o acréscimo da variável comportamento sedentário (Modelo III: $\mathrm{OR}=1,69 ; \mathrm{IC}=1,04-2,73$ ). Maior aumento da razão de chance foi observado após ajuste pela variável lanches (Modelo IV: $\mathrm{OR}=2,23$; IC=1,02 $-4,88)$. Não houve associação significativa para os adolescentes com idade $\geq 12$ anos em nenhum dos modelos apresentados.

$\mathrm{Na}$ Tabela 3 observa-se a relação entre a duração do sono e o consumo de alimentos ultraprocessados. Quanto maior a duração do sono, menor o consumo de lanches pelos adolescentes menores de 12 anos $(\beta=-49,55 ; \mathrm{IC}=-75,68$ 
Tabela 1. Descrição das variáveis sociodemográficas e presença de excesso de peso de acordo com a duração do sono de adolescentes de 10 a 14 anos de idade do município de João Pessoa-PB, 2014.

\begin{tabular}{|c|c|c|c|c|c|}
\hline \multirow{3}{*}{ Variáveis } & \multicolumn{4}{|c|}{ Duração do sono } & \multirow{3}{*}{ Total } \\
\hline & \multicolumn{2}{|c|}{$<9 \mathrm{~h}$} & \multicolumn{2}{|c|}{$\geq \mathbf{9}$} & \\
\hline & $\mathbf{n}$ & $\%$ & $\mathbf{n}$ & $\%$ & \\
\hline \multicolumn{6}{|l|}{ Sexo } \\
\hline Masculino & 195 & 30,7 & 441 & 69,3 & 636 \\
\hline Feminino & 213 & 28,5 & 535 & 71,5 & 748 \\
\hline \multicolumn{6}{|l|}{ Faixa etária } \\
\hline $10-11$ & 217 & 27,3 & 579 & 72,7 & 796 \\
\hline $12-14$ & 191 & 32,5 & 397 & 67,5 & 588 \\
\hline \multicolumn{6}{|l|}{ Escolaridade da mãe } \\
\hline Até Ensino Fundamental incompleto & 140 & 30,0 & 326 & 70,0 & 466 \\
\hline Até Ensino Médio incompleto & 96 & 29,2 & 233 & 70,8 & 329 \\
\hline Ensino Médio completo ou mais anos & 109 & 30,6 & 247 & 69,4 & 356 \\
\hline \multicolumn{6}{|l|}{ Classe econômica } \\
\hline $\mathrm{A} / \mathrm{B}$ & 120 & 29,1 & 293 & 70,9 & 413 \\
\hline $\mathrm{C} / \mathrm{D} / \mathrm{E}$ & 244 & 30,7 & 551 & 69,3 & 795 \\
\hline \multicolumn{6}{|l|}{ Turno de aula } \\
\hline Manhã/integral & 285 & 46,5 & 328 & 53,5 & 613 \\
\hline Tarde & 123 & 15,9 & 648 & 84,0 & 771 \\
\hline \multicolumn{6}{|l|}{ Excesso de peso } \\
\hline Não & 262 & 28,2 & 668 & 71,8 & 930 \\
\hline Sim & 140 & 31,5 & 304 & 68,5 & 444 \\
\hline
\end{tabular}

${ }^{\star}$ Diferença estatisticamente significativa - Teste Qui-quadrado.

Fonte: Elaborado pelos autores.

Tabela 2. Efeito da duração do sono no estado nutricional de adolescentes de acordo com a idade.

\begin{tabular}{|c|c|c|c|c|}
\hline & \multicolumn{2}{|c|}{$\begin{array}{c}<12 \text { anos de } \\
\text { idade }\end{array}$} & \multicolumn{2}{|c|}{$\begin{array}{c}\geq 12 \text { anos de } \\
\text { idade }\end{array}$} \\
\hline & OR & IC & OR & IC \\
\hline Modelo I & 1,61 & $1,01-2,55$ & 1,07 & $0,69-1,67$ \\
\hline Modelo II & 1,77 & $1,09-2,87$ & 1,12 & $0,70-1,80$ \\
\hline Modelo III & 1,69 & $1,04-2,73$ & 1,12 & $0,69-1,81$ \\
\hline Modelo IV & 2,23 & $1,02-4,88$ & 1,02 & $0,47-2,25$ \\
\hline \multicolumn{5}{|c|}{$\begin{array}{l}\text { OR: OddsRatio; IC: Intervalo de Confiança. Referência para } \\
\text { adequada duração do sono: } \geq 9 \text { horas; referência para curta } \\
\text { duração do sono: }<9 \text { horas. Modelo I: Análise bruta; Modelo } \\
\text { II: Análise ajustada por sexo, turno de aula e qualidade do } \\
\text { sono; Modelo III: Análise ajustada por sexo, turno de aula, } \\
\text { qualidade do sono e comportamento sedentário; Modelo IV: } \\
\text { Análise ajustada por sexo, turno de aula, qualidade do sono, } \\
\text { comportamento sedentário e consumo de lanches. }\end{array}$} \\
\hline
\end{tabular}

Fonte: Elaborado pelos autores.

- -23,41). Adicionando-se a variável comportamento sedentário ao modelo anterior, a associação permaneceu significativa para os adolescentes menores de 12 anos $(\beta=-49,00 ; \mathrm{IC}=-76,43$
- -21,57). No terceiro modelo, além das variáveis de confusão já utilizadas, acrescentou-se a variável atividade física, com associação também significativa $(\beta=-50,31 ; \mathrm{IC}=-77,92--22,70)$. Com relação ao consumo de lanches por adolescentes com idade maior ou igual a 12 anos, apenas mostrou-se significativa a associação quando acrescentada a variável atividade física $(\beta=-35,77$; $\mathrm{IC}=-70,56$ - -0,97). Não houve associação significativa para as bebidas açucaradas, bem como para consumo de biscoitos.

\section{Discussão}

O presente estudo analisou a relação entre a duração do sono, excesso de peso e consumo de alimentos ultraprocessados em uma amostra de adolescentes de 10 a 14 anos de idade. A média de horas de sono entre os adolescentes pesquisados foi de 9,6 horas, considerada adequada de acordo com a National Sleep Foundation ${ }^{8}$. Esse valor é maior do que aqueles observados em outros estudos no Brasil, cuja média de duração do sono variou entre $5 \mathrm{~h} 30$ e $8 \mathrm{~h}^{32-34}$. 
A rotina social dos adolescentes e seus afazeres escolares podem levar a uma redução da duração do sono, mas grande parte dos estudos analisaram a faixa etária entre 14 e $17 \operatorname{anos}^{35-38}$, com escassez de estudos que avaliaram menores de 14 anos. Nesse contexto torna-se ainda importante lembrar que o turno de aula é apontado na literatura como forte influenciador nas alterações da duração do sono de adolescentes, mostrando que estudantes do turno da manhã apresentam maior déficit de sono ${ }^{39}$. Embora vários fatores, incluindo a puberdade e demandas acadêmicas, influenciem na obtenção de um sono suficiente, a evidência defende que haja um atraso no horário de início das aulas do período da manhã, de forma a colaborar para que os adolescentes atinjam maior tempo de sono ${ }^{40,41}$, pois sabe-se que os adolescentes tendem a compensar a curta duração do sono em dias de semana com maior tempo de sono em fins de semana, o que pode influenciar na desregulação do ritmo circadiano ${ }^{40,42}$.

Zhou et al. $^{3}$ trouxeram a problemática da restrição do sono como um hábito comum entre os adolescentes na China, cuja porcentagem média de curta duração do sono foi de 34,32\% $(\mathrm{IC}=31,66$ - 36,98), sem diferença significativa entre os sexos. Hoefelmann et al. $^{43}$, em estudo realizado no Brasil, encontraram valores de curta duração ainda maiores, correspondentes a 76,7\% da amostra, porém com indivíduos de 14 a 24 anos. A tendência à maior restrição do sono com o aumento da idade pode ser atribuída à ampliação de responsabilidades e modificações de estilo de vida ao passo em que se aproximam da idade adulta $^{44}$.

O percentual de adolescentes com sobrepeso/ obesidade dos adolescentes foi de 32,3\%, valor superior ao encontrado pelo estudo ERICA ${ }^{34}$ $(25,5 \%)$. O consumo de alimentos ultraprocessados correspondeu a $31,7 \%$ da ingestão calórica total. Consumo regular e elevado de alimentos considerados não-saudáveis (variando de 18,0\% a 50,9\% do consumo total) também foram encontrados em estudo realizado por Levy et al. ${ }^{45}$ com adolescentes brasileiros. Apesar de inferior ao encontrado nas análises do Inquérito Nacional de Alimentação (INA 2008-2009), segundo o qual 54,0\% do valor energético consumido era advindo de ultraprocessados ${ }^{46}$, o valor encontrado no presente estudo merece atenção, pois acarreta em um alto consumo de sódio, gordura saturada e açúcar livre, associados ao surgimento de doenças crônicas não-transmissíveis ${ }^{47}$.

Com relação ao estado nutricional, a associação entre a curta duração do sono e a presença 
de sobrepeso/obesidade foi potencializada após ajuste pelo consumo de lanches como fator de confusão. Apesar de haver aumento da razão de chance com ajuste pelas variáveis sexo, turno de aula, qualidade do sono e comportamento sedentário, foi com ajuste a variável lanches que a $\mathrm{OR}$ mostrou-se mais elevada.

Estudo realizado por Sunwoo et al.$^{38}$ mostrou que a obesidade esteve associada à curta duração do sono, de modo que a redução da duração do sono em uma hora ocasionaria o aumento de $0,15 \mathrm{~kg} / \mathrm{m}^{2}$ no Índice de Massa Corporal (IMC) em adolescentes. Revisão sistemática realizada por Sluggett et al. ${ }^{48}$ mostrou relação existente entre a curta duração do sono e o aumento do IMC em crianças e adolescentes, de modo que quanto maior a restrição de sono maior a predisposição à obesidade.

A falta de associação entre a curta duração do sono e o excesso de peso dos adolescentes mais velhos condiz com os resultados sugeridos por Lytle et al. ${ }^{49}$, em que a partir de uma coorte de 723 adolescentes, os autores não observaram a existência de relações longitudinais estatisticamente significativas para ambos os sexos. No Estudo ERICA, realizado com adolescentes brasileiros, sono e excesso de peso não apresentaram associação significativa ${ }^{34}$.

Storfer-Isser et al. ${ }^{50}$, por meio de estudo longitudinal, mostraram associação entre a curta duração do sono e o excesso de peso em meninos, porém quanto mais velhos os adolescentes mais as associações mostraram-se atenuadas. Uma possível explicação está na fase puberal em que se encontram os adolescentes mais novos. Durante a maturação sexual há uma maior taxa metabólica basal e aumento da massa muscular, o que pode tornar mais brandos os efeitos da curta duração do sono nas mudanças de estado nutricional antropométrico na adolescência. O presente estudo não mensurou o estágio de maturação sexual dos adolescentes, porém foi o grupo com idade menor que 12 anos que apresentou associação entre curta duração do sono e excesso de peso.

Atividades que constituem o comportamento sedentário, como o uso de eletrônicos, estão relacionadas ao aumento do IMC devido ao maior consumo calórico e menor gasto energético ${ }^{48}$. Nesse sentido, no presente estudo, quando utilizado como variável de confusão, o comportamento sedentário tornou mais significativa a associação entre curta duração do sono e estado nutricional dos adolescentes menores de 12 anos. No estudo PeNSE, realizado com adolescentes brasileiros, quanto maior o tempo em comporta- mento sedentário maior o consumo de alimentos ultraprocessados ${ }^{51}$.

Estudo realizado por Ferranti et al..$^{52}$ mostrou correlação inversa entre duração do sono e índice de massa corporal, massa gorda e circunferência da cintura. Revisão sistemática realizada a partir de estudos prospectivos e transversais, Shochat et al..$^{53}$ mostraram que a relação entre sono e estado nutricional de adolescentes foi atenuada após controle das variáveis, havendo relação mais forte com variáveis psicossociais do que físicas.

Algumas mudanças hormonais estão associadas ao sobrepeso e obesidade como desfecho quando tem-se a curta duração do sono como rotina. Tais mudanças envolvem hormônios como cortisol, grelina e leptina, responsáveis pelo controle da fome e da saciedade ${ }^{54}$. A restrição de sono leva a modificações nas respostas cerebrais ligadas à alimentação, com maior consumo de alimentos como mecanismo de recompensa ${ }^{55}$. No presente estudo a curta duração do sono está associada de forma significativa ao consumo de lanches para menores de 12 anos.

É interessante notar que o consumo dos alimentos classificados no grupo dos "lanches" foi associado significativamente a curta duração do sono, enquanto o grupo "biscoitos' e "bebidas açucaradas" não mostrou associação. Não só o tipo de alimento consumido, como também o horário ou refeição em que ocorreu o consumo parece ter influenciado essa associação, pois quando analisadas a presença desses alimentos em separado nas refeições dos adolescentes, observou-se que os biscoitos foram consumidos predominantemente no horário do lanche da tarde, assim como as bebidas açucaradas, que também tiveram sua frequência distribuída entre lanche da tarde e almoço. Para o grupo dos lanches, em sua maioria os alimentos foram consumidos como jantar ou lanche da noite. Sugerese que acordados até mais tarde, os adolescentes buscam os alimentos deste grupo.

Segundo Widome et al. ${ }^{56}$, a curta duração do sono parece estar associada a comportamentos alimentares menos saudáveis em adolescentes, de modo que quanto maior a restrição de horas de sono, maior o consumo destes. Os achados podem sugerir que o ritmo circadiano possui influência na alimentação e que um comportamento alimentar positivo depende de uma duração de sono alinhada ao relógio biológico do adolescente ${ }^{57}$. Estudo realizado por Kracht et al. ${ }^{58}$ mostrou que quanto maior a duração do sono dos adolescentes menor o desejo por alimentos doces e gordurosos. 
O presente estudo possui vários pontos positivos, mas também limitações. Como pontos positivos, destaca-se o fato de ser o primeiro estudo brasileiro a buscar a relação entre a duração do sono e o estado nutricional antropométrico de adolescentes por meio da análise de grupos alimentares, com foco nos alimentos ultraprocessados. Além disso, trata-se de uma amostra representativa da população de adolescentes de escolas públicas em uma cidade do Nordeste do país.

O presente estudo utilizou o grupo de alimentos ultraprocessados por entender que na fase da adolescência é mais importante a avaliação em torno do tipo de alimento consumido de forma ampla e completa, e não apenas dos componentes desses alimentos, ou de suas calorias, de forma genérica ${ }^{57}$.

Com relação ao ponto de corte referente à curta duração do sono, estudos da literatura utilizam diferentes valores para classificação, o que pode ser um viés ao comparar resultados. O presente estudo utilizou a classificação mais atual para a faixa etária em questão ${ }^{8}$.

Quanto às limitações, trata-se de um estudo transversal, que não permite a inferência da relação causa-efeito ao longo do tempo. O presente estudo tratou apenas de duração do sono e não de sua variabilidade. O questionário aplicado não permitiu a avaliação de dados relativos ao início do sono e dificuldades para despertar, bem como presença de problemas relacionados ao sono, como a insônia, nem individualidades de cada adolescente, como o cronótipo e a rotina social. A ausência da variável maturação sexual é uma limitação, pois a mesma poderia atuar com efeito moderador, influenciando o desfecho ${ }^{59}$.

$\mathrm{O}$ autorrelato das informações referentes ao sono não deve ser considerado uma fonte de viés, pois apresentam validade comprovada quando comparados com métodos que utilizam acelerometria para captação de movimentos do corpo a fim de detectar alterações do sono e do ritmo circadiano $^{42,60}$. Questionários autorreferidos para mensuração dos níveis de atividade física em adolescentes também já foram utilizados como critério de referência em outros estudos e são ferramentas válidas para coleta desse tipo de informações ${ }^{61,62}$.

É fundamental que os responsáveis pelas crianças e adolescentes, bem como os profissionais de saúde estejam cientes da importância do sono nessas fases da vida ${ }^{63}$. São necessárias pesquisas que desenvolvam intervenções eficazes para melhoria efetiva da duração do sono e redução do risco de obesidade em crianças e adolescentes ${ }^{48}$.

\section{Conclusão}

Foi observada associação entre a curta duração do sono e o excesso de peso em adolescentes menores de 12 anos, mas não entre aqueles com 12 anos ou mais. Observou-se ainda associação entre o maior consumo de lanches e a curta duração do sono para menores de 12 anos, sendo existente a associação em adolescentes mais velhos apenas quando a variável atividade física foi utilizada como ajuste.

Diversos estudos mostram a curta duração do sono como um importante influenciador do surgimento de problemas comportamentais e de saúde durante a adolescência, porém poucos estudos analisam a fase inicial da adolescência, bem como o consumo de alimentos ultraprocessados e sua relação com a restrição de sono.

Os resultados do estudo trazem importantes reflexões sobre a relação entre duração do sono, obesidade e consumo de alimentos ultraprocessados. Os dados podem fornecer importantes informações de saúde pública sobre as relações entre estilo de vida e saúde de adolescentes, fundamentais para o desenvolvimento de intervenções, estratégias e programas educacionais voltados a essa fase da vida.

Tais achados reforçam a necessidade de que adolescentes apresentem rotinas saudáveis de duração do sono e qualidade alimentar, de forma a evitar o risco de obesidade e outros problemas de saúde ligados ao estilo de vida. São necessários mais estudos que possibilitem a análise das relações causais entre sono, estado nutricional e alimentação de adolescentes. 


\section{Colaboradores}

EVO Santos trabalhou na concepção, redação, design do artigo, análise e interpretação dos dados. ATC Almeida contribuiu com a análise e interpretação dos dados. FELL Ferreira trabalhou na concepção, redação, análise dos dados e revisão do texto final.

\section{Referências}

1. Carskadon MA, Dement WC. Chapter 2 - Normal human sleep: an overview. In: Kryger MH, Roth T, Dement WC, editores. Principles and practice of sleep medicine. St. Louis: Elsevier Saunders; 2005. p. 13-23.

2. Del Ciampo LA. O sono na adolescência. Adolesc Saude 2012; 9(2):60-66.

3. Zhou HQ, Shi WB, Wang XF, Yao M, Cheng GY, Chen PY, Li DG. An epidemiological study of sleep quality in adolescents in South China: a school-based study. Child Care Health Dev 2012; 38(4):581-587.

4. Guo X, Zheng L, Li Y, Yu S, Sun G, Yang H, Zhou X, Zhang X, Sun Z, Sun Y. Differences in lifestyle behaviors, dietary habits, and familial factors among normal-weight, overweight, and obese Chinese children and adolescentes. Int J Behav Nutr Phy 2012; 9:120.

5. Jong E, Stocks T, Visscher TLS, HiraSing RA, Seidell JC, Renders CM. Association between sleep duration and overweight: the importance of parenting. Int $J$ Obes 2012; 36(10):1278-1284.

6. Martinez SM, Tschann JM, Greenspan LC, Deardorff J, Penilla C, Flores E, Pasch LA, Gregorich SF, Butte NF. Is it time for bed? Short sleep duration increases risk of obesity in Mexican American children. Sleep Med 2014; 15(12):1484-1489.

7. Brand S, Kirov R. Sleep and its importance in adolescence and in common adolescent somatic and psychiatric conditions. Int J Gen Med 2011; 4:425-442.

8. National Sleep Foundation. Recommends New Sleep Times [Internet]. 2015 [acessado 2015 mar 15]. Disponível em: https://sleepfoundation.org/media-center/press-release/national-sleep-foundation-recommends-new-sleep-times/.

9. Guidolin M, Gradisar M. Is shortened sleep duration a risk factor for overweight and obesity during adolescence? A review of the empirical literature. Sleep Med 2012; 13(7):779-786.

10. Ruiz N, Rangel A, Rodriguez C, Rodriguez L, Rodriguez $\mathrm{V}$. Relationship among nocturnal sleep deficit, excess weight and metabolic alterations in adolescents. Arch Argent Pediatr 2014; 112(6):511-518.

11. He F, Bixler EO, Liao J, Berg A, Kawasawa YI, Fernandez Mendoza J, Vgontzas AN, Liao D. Habitual sleep variability, mediated by nutrition intake, is associated with abdominal obesity in adolescents. Sleep Med 2015; 16(12):1489-1494.

12. Pileggi C, Lolito F, Bianco A, Nobile CG, Paiva M. Relationship between Chronic Short Sleep Duration and Childhood Body Mass Index: A School-Based CrossSectional Study. PLoS One 2013; 8(6):e66680.

13. Weiss A, Xu F, Storfer-Isser A, Thpmas A. Levers-Landis CE, Redline S. The Association of Sleep Duration with Adolescents' Fat and Carbohydrate Consumption. Sleep 2010; 33(9):1201-1209.

14. Córdova FV, Barja S, Brockmann PE. Consequências da curta duração do sono na ingestão alimentar de crianças: uma revisão sistemática e metanálise. Rev Med Sono 2018; 42:68-84.

15. Crispim CA, Zalcman I, Dáttilo M, Padilha HVG, Tufik S, Mello MTD. Relação entre sono e obesidade: uma revisão da literatura. Arq Bras Endocrinol Metab 2007; 51(7):1041-1049. 
16. Hart CN, Cairns A, Jelalian E. Sleep and obesity in children and adolescents. Pediatr Clin North Am 2011; 58(3):715-733.

17. Bel S, Michels N, De Vriendt T, Patterson E, Cuenca-García M, Diethelm K, Gutin B, Grammatikaki E, Yannis M, Leclercq C, Ortega FB, Moreno LA, Gottrand F, Gonzales-Gross M, Widhalm K, Kafatos A, Garaulet M, Molnar D, Kaufmamn J-M, Gilbert CC, Hallstrom L, Sjostrom M, Marcos A, Henauw SD, Huybrechts I. Association between self-reported sleep duration and dietary quality in European adolescents. Br J Nutr 2013; 110(5):949-959.

18. Chiarelli G, Ulbrich AZ, Bertin RL. Body composition and dietary intake of adolescents from public schools in Blumenau (Brazil). Rev Bras Cineatropom Desemp Hum 2011; 13(4):265-271.

19. Associação Brasileira de Empresas de Pesquisa (ABEP). Critério de classificação econômica Brasil, 2015 [Internet]. [acessado 2015 mar 15]. Disponível em: http://www.abep.org/.

20. Garaulet M, Ortega FB, Ruiz JR, Rey-Lopez JP, Beghin L, Manios, Cuenca-Garcí M, Plada M, Diethelm K, Kafatos A, Molna'r D, Al-Tahan J, Moreno LA. Short sleep duration is associated with increased obesity markers in European adolescents: effect of physical activity and dietary habits. The HELENA study. Int $J$ Obes 2011; 35(10):1308.

21. American Academy of Pediatrics. Children, adolescents, and the media. Pediatrics 2013; 132(5):958-961.

22. Farias Júnior JCD, Lopes ADS, Mota J, Santos MP, Ribeiro JC, Hallal PC. Validade e reprodutibilidade de um questionário para medida de atividade física em adolescentes: uma adaptação do Self-Administered Physical Activity Checklist. Rev Bras Epidemiol 2012; 15:198-210.

23. World Health Organization (WHO). Global recommendations on physical activity for health. Geneva: WHO; 2010.

24. Lohman TG, Roche AF, Martorell R. Anthropometric standardization reference manual. Champaign: $\mathrm{Hu}-$ man Kinetics Books; 1988.

25. Brasil. Ministério da Saúde (MS). Secretaria de Atenção à Saúde. Departamento de Atenção Básica. Orientações para a coleta e análise de dados antropométricos em serviços de saúde: norma técnica do Sistema de Vigilância Alimentar e Nutricional-SISVAN. Brasília: MS; 2011.

26. Verly-Jr E, Sichieri R, Baltar VT. Correção de medidas de associação pela variação do dia a dia no consumo alimentar: avaliação do desempenho por meio de simulação. Cad Saude Publica 2017; 33(6):e00173216.

27. Moshfegh AJ, Rhodes DG, Baer DJ, Murayi T, Clemens JC, Rumpler WV, Paul DR, Sebastian RS, Kuczynski KJ, Ingwersen LA, Staples RC, Cleveland LE. The US Department of Agriculture Automated Multiple-Pass Method reduces bias in the collection of energy intakes. Am J Clin Nutr 2008; 88(2):324-332.

28. Zabotto CB, Viana RPDT, Gil MDF. Registro fotográfico para inqueritos dietéticos: utensilios e porçöes. Goiânia: UFG; 1996.

29. Monteiro CA, Cannon G, Moubarac JC, Levy RB, Louzada MLC, Jaime PC. The UN Decade of Nutrition, the NOVA food classification and the trouble with ultra-processing. Public Health Nutr 2018; 21(1):5-17.
30. Monteiro CA, Cannon G, Levy RB, Moubarac JC, Louzada ML, Rauber F, Baraldi LG. Ultra-processed foods: what they are and how to identify them. Public Health Nutr 2019; 22(5):936-941.

31. Harttig U, Haubrock J, Knüppel S, Boeing H. The MSM program: web-based statistics package for estimating usual dietary intake using the Multiple Source Method. Eur J Clin Nutr 2011; 65(S1):S87.

32. Pereira EF, Barbosa DG, Andrade RD, Claumann GS, Pelegrini A, Louzada FM. Sono e adolescência: quantas horas os adolescentes precisam dormir? J Bras Psiquiatr 2015; 64(1):40-44.

33. Rombaldi AJ, Soares DG. Indicadores da prática de atividade física e da qualidade do sono em escolares adolescentes. Rev Bras Cien Esporte 2016; 38(3):290296.

34. Oliveira G, Silva TLND, Silva IBD, Coutinho ESF, Bloch KV, Oliveira ERAD. Agregação dos fatores de risco cardiovascular: álcool, fumo, excesso de peso e sono de curta duração em adolescentes do estudo ERICA. Cad Saude Publica 2019; 35:e00223318.

35. Barbosa Filho VC, Silva KS, Rech CR, Brito ALS, Oliveira ESAD, Nahas, MV. Changes in lifestyle and self -rated health among high school students: A prospective analysis of the "Saúde na Boa" project. Rev Bras Cineantropom Desemp Hum 2014; 16:55-67.

36. Araújo J, Severo M, Ramos, E. Sleep duration and adiposity during adolescence. Pediatrics 2012; 130(5):e1146-e1154.

37. Schäfer AA, Domingues MR, Dahly DL, Meller FO, Gonçalves H, Wehrmeister FC, Assunção MCF. Sleep duration trajectories and body composition in adolescents: prospective birth cohort study. PloS One 2016; 11(3):e0152348.

38. Sunwoo JS, Yang KI, Kim JH, Koo DL, Kim D, Hong SB. Sleep Duration Rather than Sleep Timing is Associated with Obesity in Adolescents. Sleep Med 2020; 68:184-189.

39. Adolescent Sleep Working Group. School start times for adolescents. Pediatrics 2014; 134(3):642-649.

40. Lima SBDS, Ferreira-Lima W, Lima FÉB, Lima FB, Santos A, Fernandes CAM, Fuentes JP. Sleep Hours: Risk behavior in adolescents from different countries. Cien Saude Colet 2020; 25(3):957-965.

41. Grandner MA, Schopfer EA, Sands Lincoln M, Jackson N, Malhotra A. Relationship between sleep duration and body mass index depends on age. Obesity 2015; 23(12):2491-2498.

42. Kong AP, Wing YK, Choi KC, Li AM, Ko GT, Ma RC, Tong PC, Ho C-S, Chan MH, Ng MH, Lau J, Chan JC. Associations of sleep duration with obesity and serum lipid profile in children and adolescents. Sleep Med 2011; 12(7):659-665.

43. Hoefelmann LP, Silva KS, Barbosa Filho VC, Silva JAD, Nahas MV. Behaviors associated to sleep among high school students: cross-sectional and prospective analysis. Rev Bras Cineantropom Desemp Hum 2014; 16:68-78.

44. Carskadon MA, Sleep in adolescents: the perfect storm. Pediatr Clin North Am 2011; 58(3):637-647.

45. Levy RB, Castro IRRD, Cardoso LDO, Tavares LF, Sardinha LMV, Gomes FDS, Costa AWND. Consumo e comportamento alimentar entre adolescentes brasileiros: Pesquisa Nacional de Saúde do Escolar (PeNSE), 2009. Cien Saude Colet 2010; 15(Supl. 2):3085-3097. 
46. Pereira RA, Duffey KJ, Sichieri R, Popkin BM. Sources of excessive saturated fat, trans fat and sugar consumption in Brazil: an analysis of the first Brazilian nationwide individual dietary survey. Public Health Nutr 2014; 17(1):113-121.

47. Souza ADM, Barufaldi LA, Abreu GDA, Gainnini DT, Oliveira CLD, Santos MMD, Leal VS, Vasconcelos FAG. ERICA: ingestão de macro e micronutrientes em adolescentes brasileiros. Rev Saude Publica 2016; 50:5s

48. Sluggett L, Wagner SL, Harris RL. Sleep duration and obesity in children and adolescents. Can J Diabetes 2019; 43(2):146-152.

49. Lytle LA, Pasch KE, Farbakhsh K. The relationship between sleep and weight in a sample of adolescents. Obesity 2011; 19(2):324-331.

50. Storfer-Isser A, Patel SR, Babineau DC, Redline S. Relation between sleep duration and BMI varies by age and sex in youth age 8-19. Pediatr Obes 2012; 7(1):53-64.

51. Costa CDS, Flores TR, Wendt A, Neves RG, Assunção MCF, Santos IS. Comportamento sedentário e consumo de alimentos ultraprocessados entre adolescentes brasileiros: Pesquisa Nacional de Saúde do Escolar (PeNSE), 2015. Cad Saude Publica 2018 34:e00021017.

52. Ferranti R, Marventano S, Castellano S, Giogianni G, Nolfo F, Rametta S, Matalone M, Mistretta A. Sleep quality and duration is related with diet and obesity in young adolescent living in Sicily, Southern Italy. Sleep Sci 2016; 9(2):117-122.

53. Shochat T, Cohen-Zion, M, \&Tzischinsky, O. Functional consequences of inadequate sleep in adolescents: a systematic review. Sleep Med Rev 2014; 18(1):75-87.

54. Taheri S, Lin L, Austin D, Young T, Mignot E. Short sleep duration is associated with reduced leptin, elevated ghrelin, and increased body mass index. PLoS Med 2004; 1(3):e62.

55. Demos KE, Sweet LH, Hart CN, McCaffery JM, Williams SE, Mailloux KA, Trautvetter J, Owens MM, Wing, RR. The effects of experimental manipulation of sleep duration on neural response to food cues. Sleep 2017; 40(11):zsx125.

56. Widome R, Lenk KM, Laska MN, Erickson DJ, Iber C, Kilian G, Wahlstrom K. Sleep Duration and Weight-Related Behaviors among Adolescents. Child Obes 2019; 15(7):434-442.

57. Duraccio KM, Krietsch KN, Chardon ML, Van Dyk TR, Beebe DW. Poor sleep and adolescent obesity risk: a narrative review of potential mechanisms. Adolesc Health Med Ther 2019; 10:117.

58. Kracht CL, Chaput JP, Martin CK, Champagne CM, Katzmarzyk PT, Staiano AE. Associations of Sleep with Food Cravings, Diet, and Obesity in Adolescence. Nutrients 2019; 11(12):2899.

59. Snell EK, Adam EK, Duncan GJ. Sleep and the body mass index and overweight status of children and adolescents. Child Dev 2007; 78:309e23.

60. Timmermans M, Mackenbach JD, Charreire H, Bárdos H, Compernolle S, De Bourdeaudhuij L, Oppert JM, Rutter H, McKee M, Lakerveld J. Exploring the mediating role of energy balance-related behaviours in the association between sleep duration and obesity in European adults. The Spotlight Project. Prev Med 2017; 100:25-32.
61. Sallis JF, Saelens BE. Assessment of physical activity by self-report: status, limitations, and future directions. Res Q Exerc Sport 2000; 71(2):1-14.

62. Sirard J, Pate RR. Physical activity assessment in children and adolescents. Sports Med 2001;31(6):439-454.

63. Miller MA, Kruisbrink M, Wallace J, Ji C, Cappuccio FP. Sleep duration and incidence of obesity in infants, children, and adolescents: a systematic review and meta-analysis of prospective studies. Sleep 2018; 41(4):zsy018.
Artigo apresentado em 29/04/2020

Aprovado em 05/10/2020

Versão final apresentada em 07/10/2020

Editores-chefes: Romeu Gomes, Antônio Augusto Moura da Silva 
